\title{
A pulsed photoacoustic technique for studying red blood cell sedimentation
}

\author{
M. Kinnunen \\ Optoelectronics and Measurement Techniques Laboratory, University of Oulu, Erkki Koiso-Kanttilan katu 3, \\ P.O. BOX 4500, 90014, Finland \\ e-mail: matti.kinnunen@ee.oulu.fi
}

\begin{abstract}
This study shows the capability of a pulsed photoacoustic (PA) technique to measure red blood cell sedimentation and aggregation processes in vitro. Red blood cells are the main source of absorption in blood. The PA signal is proportional to the sample's optical absorption coefficient, and hence, dynamic changes in the sample can be monitored by analyzing the PA pulse amplitude and pulse arrival time. Optical coherence tomography (OCT) is used as a parallel method for comparison. Diluted whole blood and different concentrations of washed red blood cells were used as samples. The pulsed PA technique is suitable for monitoring changes in sedimentation velocity when dextran is added to the sample. When the measurement section with the fastest sedimentation rate was selected for analysis, a more than 10 -fold increase in the sedimentation rate, induced by dextran, was found with both the PA and OCT techniques. The PA pulse delay was found to be a more reliable measure of changes in the sample than the PA signal amplitude. (C) 2015 Samara State Aerospace University (SSAU).
\end{abstract}

Keywords: blood sedimentation; red blood cell aggregation; dextran; pulsed photoacoustic technique; optical coherence tomography.

Paper \#1990 received 2014.11.22; revised manuscript received 2015.01.20; accepted for publication 2015.01.20; published online 2015.03.28.

\section{References}

1. S. E. Bedell, and B. T. Bush, "Erythrocyte sedimentation rate. From folklore to facts," Am. J. Med. 78, 10011009 (1985).

2. T. L. Fabry, "Mechanism of erythrocyte aggregation and sedimentation," Blood 70(5), 1572-1576 (1987).

3. A. V. Priezzhev, N. F. Nikolai, and J. Lademann, "Light backscattering diagnostics of red blood cell aggregation in whole blood samples," Chapter 11 in Handbook of optical biomedical diagnostics, Tuchin V. V. Ed., SPIE Press, Bellingham (2002).

4. B. Neu, R. Wendy, and H. J. Meiselman, "Effects of dextran molecular weight on red blood cell aggregation," Biophys. J. 95, 3059-3065 (2008).

5. A. Westergren, "Studies of the suspension stability of the blood in pulmonary tuberculosis," Acta Medica Scandinavica 54, 247-282 (1921).

6. L. E. Böttiger, and C. A. Svedberg, "Normal erythrocyte sedimentation rate and age," Brit. Med. J. 2, 85-87 (1967).

7. M. L. Bridgen, "Clinical utility of the erythrocyte sedimentation rate," Am. Fam. Physician 60(5), 1443-1450 (1999).

8. J. M. Jou, S. M. Lewis, C. Briggs, S.-H. Lee, B. de la Salle, and S. McFadden, "ICSH review of the measurement of the erythrocyte sedimentation rate," Int. Jnl. Lab. Hem. 33, 125-132 (2011).

9. A. V. Priezzhev, O. M. Ryaboshapka, N. N. Firsov, and I. V. Sirko, "Aggregation and disaggregation of erythrocytes in whole blood: study by backscattering technique," J. Biomed. Opt. 4(1), 76-84 (1999).

10. C. V. L. Pop, and S. Neamtu, "Aggregation of red blood cells in suspension: study by light-scattering technique at small angles,” J. Biomed. Opt. 13(4), 041308 (2008). 
11. X. Xu, L. Yu, and Z. Chen, "Velocity variation assessment of red blood cell aggregation with spectral domain Doppler optical coherence tomography," Ann. Biomed. Eng. 38, 3210-3217 (2010).

12. F. T. H. Yu, and G. Cloutier, "Experimental ultrasound characterization of red blood cell aggregation using the structure factor size estimator (SFSE)," J. Acoust. Soc. Am. 122, 645-656 (2007).

13. E. Franceschini, F. T. H. Yu, F. Destrempes, and G. Cloutier, "Ultrasound characterization of red blood cell aggregation with interventing attenuation tissue mimicking phantoms using structure factor size and attenuation estimator," J. Acoustic Soc. Am. 127, 1104-1115 (2010).

14. S. J. Lee, H. Ha, and K.-H. Nam, "Measurement of red blood cell aggregation using X-ray phase contrast imaging," Opt. Express 18, 26052-26061 (2010).

15. Z. Zhao, X. Wang, and J. F. Stoltz, "Comparision of three optical methods to study erythrocyte aggregation," Clinical Hemorheology and Microcirculation 21, 297-302 (1999).

16. M. R. Hardeman, J. G. G. Dobbe, and C. Ince, "The laser-assisted optical rotational cell analyzer (LORCA) as red blood cell aggregometer," Clinical Hemorheology and Microcirculation 25, 1-11 (2001).

17. I. Y. Kuo, and K. K. Shung, "High frequency ultrasonic backscatter from erythrocyte suspension," IEEE Trans. Biomed. Eng. 41(1), 29-34 (1994).

18. M. S. van der Heiden, M. G. M. de Kroon, N. Bom, and C. Borst, "Ultrasound backscatter at $30 \mathrm{MHz}$ from human blood: influence of rouleau size affected by blood modification and shear rate," Ultrasound in Med. \& Biol. 21(6), 817-826 (1995).

19. A. A. Oraevsky, S. L. Jacques, F. K. Tittel, "Measurement of tissue optical properties by time-resolved detection of laser-induced transient stress," Appl. Opt. 36, 402-415 (1997).

20. J. S. Antoniow, J. Egèe, M. Chirtoc, C. Bissieux, and G. Potron, "Real time analysis of erythrocyte sedimentation by photothermal methods," Journal de Physique IV, CT-469 - C7-472 (1994).

21. A. Landa, and J. J. Alvarado-Gil, "Photoacoustic monitoring of real time blood and hemolymph sedimentation," Rev. Sci. Instrum. 74(1), 377-379 (2003).

22. P. Helander, and I. Lundström, "Whole blood - a sedimenting sample studied by photoacoustic spectroscopy," J. Photoacoust. 1(2), 203-215 (1982).

23. S. Oka, "A physical theory of erythrocyte sedimentation," Biorheology 22, 315-321 (1985).

24. E. I. Galanzha, and V. P. Zharov, "In vivo photoacoustic and photothermal cytometry for monitoring multiple blood rheology parameters," Cytometry Part A 79 A, 746-757 (2011).

25. H. Schmid-Schönbein, H. Malotta, and F. Striesow, "Erythrocyte aggregation: causes, consequence and methods of assessment," J. Netherlands Soc. Clin. Chem. 15, 88-97 (1990).

26. J. K. Armstrong, R. B. Wendy, H. J. Meiselman, and T. C. Fisher, "The hydrodynamic radii of macromolecules and their effect on red blood cell aggregation," Biophys. J. 87, 4259-4270 (2004).

27. X. Xu, R. K. Wang, J. B. Elder, and V. V. Tuchin, "Effect of dextran-induced changes in refractive index and aggregation on optical properties of whole blood," Phys. Med. Biol. 48, 1205-1221 (2003).

28. V. V. Tuchin, X. Xu, and R. K. Wang, "Dynamic optical coherence tomography in studies of optical clearing, sedimentation, and aggregation of immersed blood," Appl. Opt. 41(1), 258-271 (2002).

29. V. V. Tuchin, R. K. Wang, E. I. Galanzha, J. B. Elder, and D. M. Zhestkov, "Monitoring of glycated hemoglobin by OCT measurements of refractive index," Proc. SPIE 5316, 66-77 (2004).

30. X. Xu, V. V. Tuchin, and R. K. Wang, "Immersion technique as a tool for in depth OCT imaging trough human blood and body's interior tissues," Proc. SPIE 4251, 89-96 (2001).

31. V. V. Tuchin, R. K. Wang, and X. Xu, "Whole blood and RBC sedimentation and aggregation study using OCT," Proc. SPIE 4263, 143-149 (2001).

32. X. Xu, L. Yu, and Z. Chen, "Effect of erythrocyte aggregation on hematocrit measurement using spectraldomain optical coherence tomography," IEEE Trans. Biomed. Eng. 55(12), 2753-2758 (2008).

33. V. V. Tuchin, Optical clearing of tissues and blood, SPIE press, Washington, Bellingham, USA, 2006.

34. M. Yu Kirillin, A. V. Priezzhev, V. V. Tuchin, R. K. Wang, and R. Myllylä, "Effect of red blood cell aggregation and sedimentation on optical coherence tomography signals from blood samples," J. Phys. D: Appl. Phys. 38, 2582-2589 (2005).

35. V. V. Tuchin, D. M. Zhestkov, A. N. Bashkatov, and E. A. Genina, "Theoretical study of immersion optical clearing of blood in vessels at local hemolysis," Opt. Express 12(13), 2966-2971 (2004).

36. C. Li, and L. V. Wang, "Photoacoustic tomography and sensing in biomedicine," Phys. Med. Biol. 54, R59R97 (2009).

37. J. Laufer, C. Elwell, D. Delpy, and P. Beard, "In vitro measurements of absolute blood oxygen saturation using pulsed near-infrared photoacoustic spectroscopy: accuracy and resolution,” Phys. Med. Biol. 50, 4409-4428 (2005).

38. J. Laufer, D. Delpy, C. Elwell, and P. Beard, "Quantitative spatially resolved measurement of tissue chromophore concentrations using photoacoustic spectroscopy: application to the measurement of blood oxygenation and haemoglobin concentration," Phys. Med. Biol. 52, 141-168 (2007). 
39. R. K. Saha, and M. C. Kolios, "A simulation study on photoacoustic signals from red blood cells," J. Acoust. Soc. Am. 129(5), 2935-2943 (2011).

40. E. Hysi, R. K. Saha and M. C. Kolios, "On the potential of using photoacoustic spectroscopy to monitor red blood cell aggregation,” Proc. SPIE 8222, 82220Q (2012).

41. G. Barshtein, I. Tamir, and S. Yedgar, "Red blood cell rouleaux formation in dextran solution: dependence on polymer conformation," Eur. Biophys. J. 27, 177-181 (1998).

42. M. Kinnunen, and R. Myllylä, "Application of optical coherence tomography, pulsed photoacoustic technique and time-of-flight technique to detect changes in the scattering properties of a tissue-simulating phantom," J. Biomed. Opt. 13(2), 024005 (2008).

43. G. J. Diebold, and T. Sun, "Properties of photoacoustic waves in one, two, and three dimensions," Acustica 80, 339-351 (1994).

44. M. Kinnunen, "Monitoring the effect of dextran on blood sedimentation using a pulsed photoacoustic technique," Proc. SPIE 8222, 82221E (2012).

45. F. A. Duck, Physical properties of tissue: a comprehensive reference book, Academic Press Inc., San Diego (1990).

46. M. Meinke, G. Müller, J. Helfmann, and M. Friebel, "Optical properties of platelets and blood plasma and their influence on the optical behavior of whole blood in the visible to near infrared wavelength range," J. Biomed. Opt. 12(1), 014024 (2007).

47. A. B. Karpiouk, S. R. Aglyamov, S. Mallidi, J. Shah, W. G. Scott, J. M. Rubin, and S. Y. Emelianov, "Combined ultrasound and photoacoustic imaging to detect and stage deep vein thrombosis: phantom and ex vivo studies," J. Biomed. Opt. 13(5), 054061 (2008).

48. R. D. Eastham, "The erythrocyte sedimentation rate and the plasma viscosity," J. Clin. Path. 7, 164-167 (1954).

49. S. Kim, S. Yang, and D. Lim, "Effect of dextran on rheological properties of rat blood," J. Mech. Sci. Technol. 23, 868-873 (2009).

50. M. Kinnunen, Z. Zhao, and R. Myllylä, "Glucose-induced changes in the optical properties of Intralipid," Opt. Spectrosc. 101(1), 54-59 (2006).

51. B. E. Treeby, E. Z. Zhang, A. S. Thomas, and B. T. Cox, "Measurement of the ultrasound attenuation and dispersion in whole human blood and its components from 0-70 MHz," Ultrasound in Med. \& Biol. 37(2), 289-300 (2011).

52. H.-J. Lim, Y.-J. Lee, J.-H. Nam, S. Chung, and S. Shin, “Temperature-dependent threshold shear stress of red blood cell aggregation," J. Biomech. 43, 546-550 (2010).

53. D. J. Faber, F. J. van der Meer, M. C. G. Aalders, and T. G. van Leeuwen, "Quantitative measurement of attenuation coefficients of weakly scattering media using optical coherence tomography," Opt. Express 12(19), 4353-4365 (2004).

54. Z. Zhao, S. Nissilä, O. Ahola, and R. Myllylä, "Production and detection theory of pulsed photoacoustic wave with maximum amplitude and minimum distortion in absorbing liquid," IEEE Trans. Instrum. Meas. 47(2), 578-583 (1998).

\section{Introduction}

Red blood cell sedimentation velocity, also called erythrocyte sedimentation rate (ESR), is an indicator in clinical diagnosis and an auxiliary method for indicating possible underlying disease [1-4]. The initial method for measuring ESR in test tubes was described by Westergren [5]. The analysis of the results is not very straightforward. There are variations in the ESR values of healthy controls [6] and the ESR value may be affected by several factors in different diseases [7]. However, ESR measurement is still used as a test to screen and monitor disease processes that affect plasma proteins and the ESR value [8]. The RBC aggregation process can be altered in the case of a disease compared with healthy controls [9], which also affects the ESR value.

In vitro methods of diagnosing red blood cell aggregation include both optical and acoustical techniques, namely, backscattering optical radiation [9], small-angle light scattering [10], optical coherence tomography (OCT) [11], and ultrasonic backscattering $[12,13]$. Also other methods can be found in literature, e.g. X-ray phase contrast imaging [14]. Optical methods are based on light scattering and detection of changes in the scattered signals. Both backscattering and transmitted light can be measured $[15,16]$. They are also sensitive to changes in the refractive index. Commercial instruments facilitate laboratory analysis [15]. In the ultrasound method [12], measurement of backscattered power is dependent on the scatterer concentration. This scatterer density is high in blood, and therefore it makes ultrasonic blood characterization difficult. A nonlinear relationship has been found between backscattering power and scatterer concentration in an extremely dense medium (such as blood) [17]. Backscattering is dependent on an acoustic impedance mismatch between the tissue and cell components [12]. In blood, this means the acoustic impedance difference between RBCs and the surrounding medium. More specifically, it is 
related to hemoglobin concentration inside and outside of RBCs [18].

Illuminating a sample with short laser pulses can result in generation of acoustic waves that travel through the examined medium. The sample's optical absorption at a certain wavelength will affect the strength of the acoustic signal. Other parameters affecting the signal are the volume expansion coefficient and specific heat capacity. The PA signal generation principle differs from the ultrasonic backscattering technique, and different problems are associated with that technique than with the ultrasonic technique [19]. Earlier papers on monitoring blood sedimentation with photoacoustic and photothermal methods have used chopped light and phase-sensitive detection [20-22]. Both amplitude and phase have been analyzed. These parameters tell about the sample's properties during the sedimentation process [20-22]. Helander et al. [22] found that a lower initial RBC concentration gave a higher final amplitude value, probably due to the more closely packed structure of the RBCs at the bottom of the cuvette at the end of the experiment. Different theoretical models have been developed to understand the physics behind the sedimentation process [2,20,23]. Earlier PA studies on blood sedimentation were focused on understanding the dynamics of sedimentation and modeling the process [20-23]. On the other hand, blood rheology in vivo can be studied, e.g. by photoacoustic flow cytometry (PAFC), where rheological processes can be monitored at a single RBC level [24]. Several blood parameters, such as blood viscosity, hematocrit, and RBC aggregation affect kinetic and flow properties in vivo [24].

Non-specific adsorption of aggregating plasma proteins on the surfaces of RBCs will induce $\mathrm{RBC}$ aggregation $[9,25,26]$. RBC aggregation can also be induced with dextrans $[4,26]$. The OCT technique for characterizing blood sedimentation has been studied extensively during the last ten years. In addition, different biocompatible agents, such as dextrans, have been used to study optical clearing to enhance OCT imaging depth and light penetration [27-33]. Also, theoretical models have been developed to understand optical methods in more detail [34], as well as blood optical clearing induced by local hemolysis [35].

Recent increased utilization of the PA technique in imaging and sensing applications [19,36-38] has also raised interest towards in vitro diagnostics of blood aggregation properties with the PA method $[39,40]$. Saha et al. performed Monte Carlo simulations to study the effect of RBC aggregation on the PA signal [39], whereas Hysi et al. used a pulsed laser to induce PA waves in the sample and used the acoustic frequency spectrum to analyze RBC aggregation [40]. Galanzha and Zharov have done valuable work in the field of PAFC and blood diagnostics in vitro and in vivo [24].

The aim of this work was to explore the capability of the pulsed photoacoustic (PA) technique to monitor dextran-induced changes in the sedimentation rate in diluted whole blood as well as washed RBC samples with different hematocrit values. Here it is shown that it is possible to monitor the sedimentation process with PA pulse delays generated by absorbing optical pulses in the moving blood layer. OCT is used as a parallel method to enhance understanding of the changes in the $\mathrm{RBC}$ samples during the experiments. The results are discussed in relation to data found in literature which have been obtained with optical methods.

\section{Materials and Methods}

\subsection{Sample preparation}

Blood samples were taken from a healthy volunteer (male, 33 years). EDTA was used to prevent coagulation. Table 1 presents the ingredients of the different samples. Samples 1-4 were prepared by diluting whole blood with PBS (Thermo Scientific, HyClone Laboratories, Inc., Utah, USA). Samples 5-8 were prepared by diluting $\mathrm{RBC}$ mass with the same PBS. RBCs were separated from the whole blood with a centrifuge and washed with PBS (two times $10 \mathrm{~min}$ at 2000 rpm). Dextran T500 (Pharmacia Fine Chemicals AB, Uppsala, Sweden, molecular weight 500000) was used in the experiments to induce RBC aggregation. When washed RBCs were used, also bovine serum albumin (Sigma Chemicals CO., St. Louis, MO, USA) with a concentration of $1.0 \mathrm{~g} / \mathrm{dl} \quad(1 \%$ albumin concentration was taken from Ref. 41) was used. The experiments were repeated on two separate days with two different sets of samples (denoted as sample (18)_1 and sample (1-8)_2).

Table 1 Samples with different analytes.

\begin{tabular}{ccccc}
\hline No. & Sample & $\begin{array}{c}\text { Dextran } \\
(\mathbf{g} / \mathbf{d l})\end{array}$ & $\begin{array}{c}\text { Albumin } \\
(\mathbf{g} / \mathbf{d l})\end{array}$ & $\begin{array}{c}\text { Hct } \\
(\mathbf{\%})\end{array}$ \\
\hline 1 & diluted blood & 0 & 0 & 31 \\
\hline 2 & diluted blood & 0.5 & 0 & 31 \\
\hline 3 & diluted blood & 0 & 0 & 21 \\
\hline 4 & diluted blood & 0.5 & 0 & 21 \\
\hline 5 & washed RBCs & 0 & 0 & 27 \\
\hline 6 & washed RBCs & 0.5 & 1.0 & 27 \\
\hline 7 & washed RBCs & 0 & 0 & 18 \\
\hline 8 & washed RBCs & 0.5 & 1.0 & 18 \\
\hline & & & &
\end{tabular}

\subsection{Measurement setup}

Fig. 1 shows a schematic diagram of a measurement setup. A diode-pumped solid state Nd:YAG laser with a passive Q-switched crystal (model LCS-DTL-324QT) at a wavelength of $1064 \mathrm{~nm}$ was used in the measurements. The pulse repetition rate of the laser was $500 \mathrm{~Hz}$ and the pulse length was $<10 \mathrm{~ns}$. The pulse energy was $\approx 106 \mu \mathrm{J}$ before entering the optical fiber. The optical pulse was slightly focused on the sample surface after the fiber to form a spot with a diameter of 
$\sim 2 \mathrm{~mm}$. A photoacoustic transducer with a center frequency of $1 \mathrm{MHz}$ (Panametrics V103) was used to detect acoustic signals. The signals were amplified with a preamplifier (Olympus 5662) and the data were collected with a data acquisition card (National Instruments, PCI-5124). A sampling frequency of 20 $\mathrm{MHz}$ was used and the signals were averaged 500 times. A cuvette was made of grey PVC (polyvinyl chloride) plastic material. The cuvette dimensions were: thickness $5 \mathrm{~mm}$, width $25 \mathrm{~mm}$, length $40 \mathrm{~mm}$, the total volume being $5 \mathrm{ml}$. The acoustic transducer was placed under the cuvette at a $5 \mathrm{~mm}$ distance from the sample. A 1mm-thick glass plate was used on the upper side of the cuvette.

Optical coherence tomography was used to monitor blood sedimentation simultaneously with the PA experiment. A commercial OCT device (manufactured by the Institute of Applied Physics, Nizhny Novgorod, Russia) was used. The wavelength of the light source was $910 \mathrm{~nm}$, axial resolution in air $<10 \mu \mathrm{m}$, and spatial resolution $<10 \mu \mathrm{m}$ for 1-x magnification. The details of the device are described elsewhere [42].

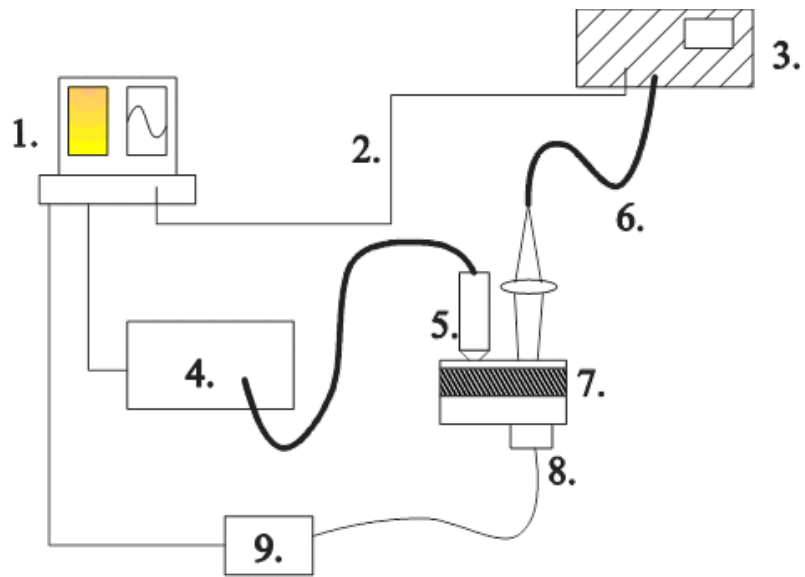

Fig. 1 Measurement setup. 1. Computer with data acquisition card and software, 2. Cable for triggering, 3. Laser source, 4. OCT device, 5. OCT probe, 6. Optical fiber illumination with laser pulses, 7. Cuvette with sample, 8. Pietzoelectric transducer, 9. Preamplifier.

\subsection{Measurement protocol}

Samples were prepared right before the measurements. The samples were prepared and the measurements were taken at room temperature. Blood and washed red blood cells were diluted to the final concentration and the sample was carefully mixed. The samples were loaded onto the cuvette and the PA and OCT measurements were started immediately. The PA signal waveform was saved on the computer at time points $0,2,4,6,8,10$, $15,20,25$, and 30 minutes. In four cases out of 16 measurements the experiment was stopped earlier than at $30 \mathrm{~min}$ due to the fast sedimentation process. The time delay of the pulse peak as well as the pulse amplitude values were extracted from the wave shapes. The OCT signal was recorded during the same period for further analysis. To visualize the sedimentation process, only a-scans were registered from a single point as a function of time (see Fig. 3). Fifty a-scans were averaged for samples without dextran and five ascans for samples with dextran. Different averaging was used because sedimentation kinetics varied considerably between the different samples. The image dimensions were $2500 \times 500$ pixels, corresponding to $550 \mu \mathrm{m} \times 1931 \mu \mathrm{m}$.

\subsection{Signal analysis}

The amplitude of the PA signal is proportional to the optical absorption coefficient $\mu_{a}$ and the Grüneisen parameter $\Gamma$, which is defined by $\beta v^{2} / C_{P}$. Eq. (1) shows this relation:

$$
\Delta \mathrm{P}=\rho v^{2} \cdot \beta \Delta \mathrm{T}=\left(\frac{\beta v^{2}}{C_{P}}\right) \cdot\left(\frac{E_{a}}{V}\right)=\Gamma H_{f} \mu_{a},
$$

where $E_{a} / V$ is the absorbed optical energy density that equals the product of laser fluence $H_{f}$ and the sample's optical absorption coefficient $\mu_{a} . \beta$ is the volume expansion coefficient, $C_{p}$ is specific heat at constant pressure, and $v$ is the velocity of acoustic waves in the medium [43]. Hence, all these parameters take part in acoustic pulse generation. The photo-induced pressure rise $\Delta \mathrm{P}$ propagates outside the irradiated volume in the form of an acoustic wave. It is worth noting that $\Gamma$ is sensitive to temperature, and temperature changes can affect the measurements $[19,36]$.

Fig. 2(a) shows examples of the PA pulse profiles from one experiment. The pulse amplitude generated in the sample (A1), the time delay $(\Delta \mathrm{t})$, and the amplitude from the bottom of the cuvette (A2) are analyzed in detail. It is clearly seen from Figs. 2(a) and (b) that the pulse amplitude increases and shifts to the earlier times (pulse delay decreases) when blood sedimentation occurs.

Sedimentation velocity can be deduced from curve 2(b) [44], $v=s / \Delta t$, where $\mathrm{s}$ is the propagation distance of the blood layer during time $\Delta \mathrm{t}$ and is the product of the pulse peak time shift and acoustic velocity. $\Delta \mathrm{t}$ is the period for that time (e.g. $10 \mathrm{~min}$ ). The pulse peak shift is in the range of microseconds and the value of $1550 \mathrm{~m} / \mathrm{s}$ is used for acoustic velocity [45].

Relative change in the PA pulse arrival time was calculated to facilitate easier comparison of the sedimentation dynamics between samples. The pulse arrival times of the experiments with different samples were divided by the arrival time of the signal at the beginning of each experiment (at time $0 \mathrm{~min}$ ). Fig. 4 shows relative values (a.u.), and values smaller than 1 indicate faster arrival of the PA pulse on the detector than at the initial stage of the experiment.

Errors in the sedimentation velocity were estimated by defining the timing error (time to save the PA signal and its deviation from full minutes) and calculating a relative error by dividing this error by the time used in velocity estimation (see Table 2). The effect of possible 
temperature variation on acoustic velocity was taken into account by adding an error of $\pm 1^{\circ} \mathrm{C}( \pm 2 \mathrm{~m} / \mathrm{s}$, see text later) in acoustic velocity. Errors were rounded up to get reasonable error estimation together with the extracted result (see Table 2).
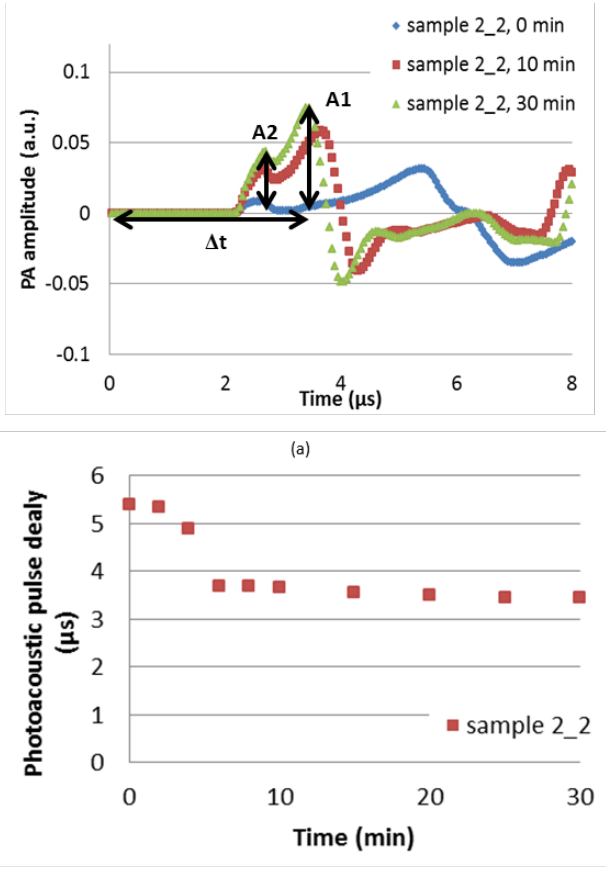

(b)

Fig. 2 (a) Photoacoustic signals at different time points of the experiment when measuring sample 2_2 and (b) the time delay of the PA pulse as a function of the measurement time.

The OCT images were analyzed by selecting averaged a-scans from two points in the image and recording the time position of those a-scans (Fig. 3). The velocity estimation range was selected so that maximal velocity could be found. Time dependency of the sedimentation velocity was shown by Antoniow et al. [20]. Then the depth profiles were plotted and the change in the depth of the blood-plasma/PBS layer was calculated. The following values for the refractive index of plasma and PBS were used in the calculations: $\mathrm{n}_{\mathrm{av}}=\mathrm{X}^{*} \mathrm{n}_{\text {plasma }}+\mathrm{Y}^{*} \mathrm{n}_{\mathrm{pbs}}: \mathrm{n}_{\text {plasma }}=1.347$ [46], and $\mathrm{n}_{\mathrm{PBS}}=1.333$ [46], resulting in the following mean values for the samples' background medium: 1: $\mathrm{n}_{\mathrm{av}}=1.3414,2: \mathrm{n}_{\mathrm{av}}=1.3414, \quad 3: \mathrm{n}_{\mathrm{av}}=1.3386$, 4: $\mathrm{n}_{\mathrm{av}}=1.3386,5: \mathrm{n}_{\mathrm{av}}=1.333, \quad 6: \mathrm{n}_{\mathrm{av}}=1.333$, 7: $\mathrm{n}_{\mathrm{av}}=1.333$, and $8: \mathrm{n}_{\mathrm{av}}=1.333$. In OCT, an error of 2 pixels in reading the image was considered as experimental error in defining sedimentation velocity. The time of an averaged a-scan was calculated from the total measurement time divided by the number of averaged a-scans in the image (2500). Errors were rounded up to get reasonable error estimation together with the extracted result (see Table 2).

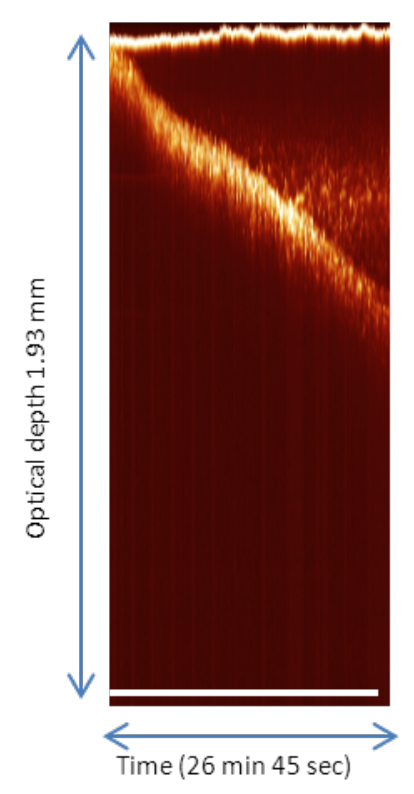

(a)

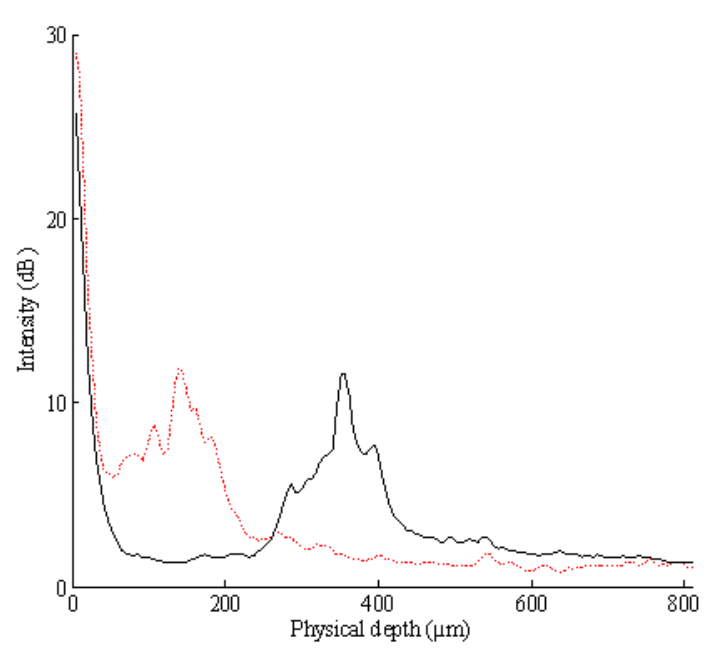

(b)

Fig. 3 (a) OCT image from sample 2_1 and (b) OCT signal depth profiles from sample $2 \_\overline{1}$ (figure (a)) at time points $64 \mathrm{~s}$ (dashed line) and $321 \mathrm{~s}$ (solid line) from the beginning of the experiments. The profiles are an average of 50 a-scans (modified from Fig. 6, Ref. [44]).

\section{Results and discussion}

Fig. 2(a) shows the PA pulse profiles from a blood sample with dextran at different time points of the experiments. The PA signal amplitude is proportional to the RBC concentration, i.e. hematocrit, and hence, to optical absorption [47]. The amplitude increases when the RBC concentration in the absorbing layer increases. Also other physical and optical properties of the sample may vary simultaneously. The PA amplitude is sensitive to changes in the contact of the transducer. Therefore, the time delay of the pressure pulse is a more reliable measure and is analyzed in more detail. Fig. 4 (a) shows 
relative changes in the PA pulse arrival time in diluted blood samples (1-4) during the sedimentation process, whereas Fig. 4(b) shows the results for washed RBC samples. The dynamics of sedimentation are clearly visible. The same can be seen in Fig. 3(a). These findings support earlier investigations [28]. The sedimentation process has non-linear behavior, which has also been found in some other publications $[2,20,23]$. It can be seen that dextran makes the sedimentation process faster by inducing $\mathrm{RBC}$ aggregation. Another phenomenon slowing down the process is the dextran-induced increase in plasma viscosity $[48,49]$. Dextran-induced increase in blood viscosity is mostly due to red blood cell aggregation [49].
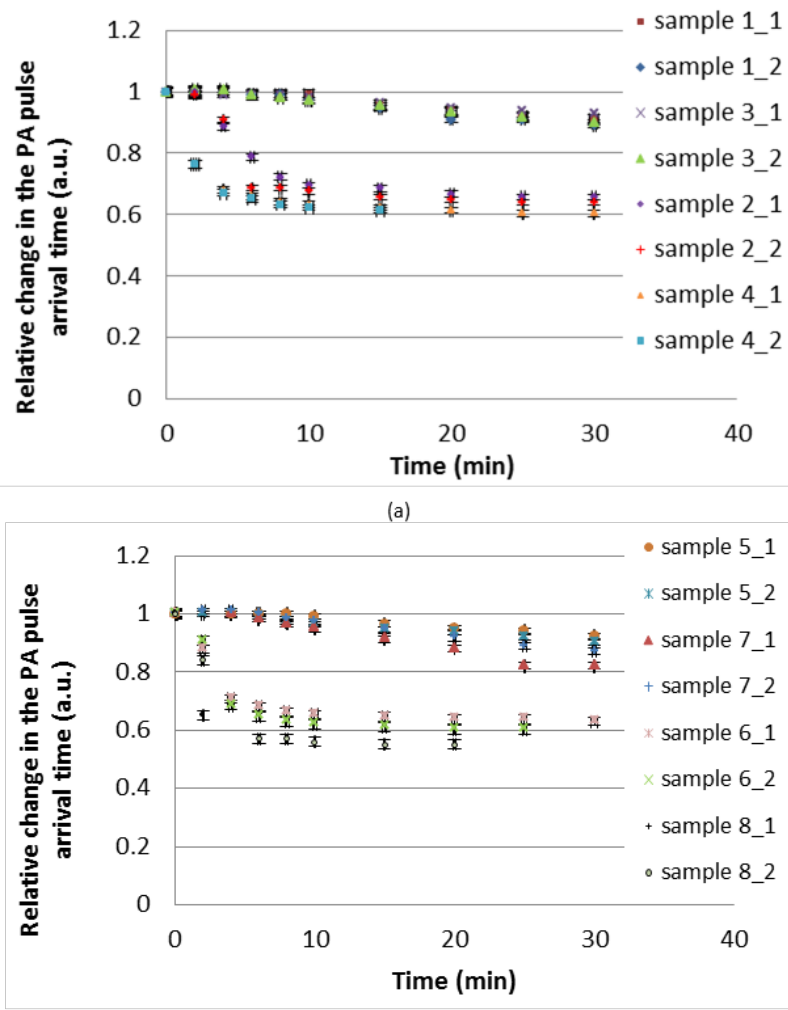

(b)

Fig. 4 (a) Relative change in the PA pulse delay as a function of the measurement time for diluted blood samples and (b) relative change in the PA pulse delay as a function of the measurement time for washed RBCs.

It is clearly seen in Fig. 2(a) that blood sedimentation increases light transmittance through the RBC layer. More light hits the cuvette, light is absorbed in the plastic and generates an acoustic wave due to thermoelastic expansion, and the PA signal amplitude (A2) from the bottom of the cuvette increases. The hematocrit of the sample affects the sedimentation rate [2]. It was found (Fig. 4(a)) that the sedimentation process was the fastest with samples 4 , where RBC concentration was the lowest.

The same effect can be seen in Fig. 4(b), where dextran increased the sedimentation rate. Due to the limited measurement time $(30 \mathrm{~min})$ and slow sedimentation velocity, the PA signal amplitudes from diluted whole blood samples remained almost constant, while dextran greatly increased the amplitude (Fig. 5).

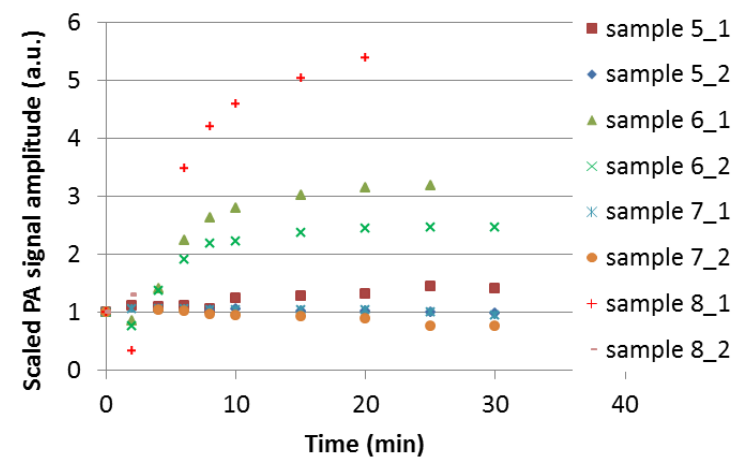

Fig. 5 Scaled PA signal amplitude as a function of the measurement time for different washed RBC samples.

Changes in the acoustic pulse delay tell about changes in the PA pulse generating area, and hence, in the sedimentation of the RBCs. The optical properties of the sample will change during $\mathrm{RBC}$ aggregation $[28,34]$. The anisotropy factor $g$ is larger for aggregates than for single cells. RBC aggregates are larger than single cells, and hence, light scattering will be more forward directed [34]. When blood sedimentation occurs, the layer with RBCs becomes thinner and the multiple scattering properties will change as the cells get closer to each other. It is also worth remembering that dextran will increase the refractive index of the sample, reducing the refractive index mismatch between RBCs and the background medium and reducing scattering (optical clearing) [27,33]. Decreased scattering increases optical energy density in the illuminated sample area [50]. Absorption also increases due to the increased cell density. These effects result in an increased PA amplitude (Fig. 5). This is also seen as increased transmittance in OCT experiments [28,30,31]. The acoustic source is hence moving down and its properties are changing due to the changing optical properties. The earlier findings with OCT [28] are now supported also by PA experiments, as shown in Figs. 4 and 5. It must be pointed out that when the background suspension of the blood sample is changed from plasma to PBS, e.g. from whole blood to washed RBCs, the refractive index of the background medium changes from 1.347 (for plasma) to 1.333 (for 0.9 saline solution or water) [46]. This alters the optical scattering properties of the sample [46]. The highest OCT signal intensity, i.e. the best image contrast, was achieved from samples 1 and 3 .

The sedimentation rate varied considerably during the experiments, especially when the aggregation process was enhanced. Hematocrit also affects sound velocity [45], being smaller for diluted concentrations. Acoustic wave velocity is dependent on both the sample's temperature and its blood hematocrit value. Sound velocity for a $1.0 \%$ saline solution of water is $1493.6 \mathrm{~m} / \mathrm{s}$ at $20^{\circ} \mathrm{C}$ and $1538.9 \mathrm{~m} / \mathrm{s}$ at $40^{\circ} \mathrm{C}$ [45]. For 
blood plasma the velocity is $1546 \mathrm{~m} / \mathrm{s}$, and for whole blood, $1584.2 \mathrm{~m} / \mathrm{s}$ (at $37{ }^{\circ} \mathrm{C}$ ) [45]. It was found that the temperature dependence of the acoustic velocity of blood is $-2 \mathrm{~m} / \mathrm{s} /{ }^{\circ} \mathrm{C}$. This value was used in error estimation, giving $0.13 \%$ error. Hence, for $20{ }^{\circ} \mathrm{C}$ the value is $1550 \mathrm{~m} / \mathrm{s}$. Another source gives a value of 1535 $\mathrm{m} / \mathrm{s}$ for PBS, $1553 \mathrm{~m} / \mathrm{s}$ for plasma, and $1590 \mathrm{~m} / \mathrm{s}$ for whole blood at $37{ }^{\circ} \mathrm{C}$ [51]. Variation in sound velocity in different samples may induce a small error in the estimated sedimentation values. It is also worth noting that viscous properties are different for plasma and PBS [46]. Viscosity is also dependent on temperature, being higher at room temperature than at $37{ }^{\circ} \mathrm{C}$ [52]. Dextran will also increase viscosity [48,49], reducing the sedimentation rate at high dextran concentrations [48]. Hence, the effects of viscosity must be taken into account when analyzing dextran-induced aggregation and enhanced sedimentation rate [48].

Table 2 shows velocity values extracted from both PA signals and OCT images. The velocity estimation range was selected so that maximal velocity could be found from the measured data. The time-dependent change in the sedimentation velocity is found in the work of Antoniov et al. [20]. Dextran accelerated the sedimentation velocity more than 10 -fold. In some cases the effect of dextran was found to be higher in samples with lower hematocrit. When the sedimentation rates for diluted blood (with plasma) and RBCs in PBS were compared, the results resembled each other considerably. The values in Table 2 for samples without dextran match well with typical values found in the literature, which are $0-15 \mathrm{~mm} / \mathrm{h}$ for men and $0-20$ $\mathrm{mm} / \mathrm{h}$ for women $[6,28]$. The plasma proteins of whole blood induce a certain amount of RBC aggregation also in healthy blood samples, which might affect the variation in sedimentation times between different persons [26].

Sources of error in the experiments are the accuracy of saving the wave shape during the experiments $(0.11-$ $6.67 \%$ ) and sample preparation accuracy. The wave shape might also be affected by the transducer's bandwidth, which makes the analysis of the pulse shape inaccurate. Time-resolved detection of PA signals with a wide frequency bandwidth transducer will be a part of future research. Figs. 4 (a) and (b) also show that samples 4 and 8 exhibit very rapid sedimentation. Therefore, a reliable value for the sedimentation rate could not be extracted for sample 1_8 (see Table 2). Hence, real-time data acquisition would be better, and the software will be developed in the future.

Sample-to-sample variation in sedimentation kinetics affects repeatability. Velocity differences obtained with OCT and PA methods might be explained by the different depths used in the analysis. In the current measurement procedure, the time point during the measurement can be more accurately determined from OCT images than from PA pulses (error in Table 2 ). In addition, the OCT signal's focus point in the sample affects signal intensity and image contrast [53]. Low image contrast makes estimation of sedimentation velocity difficult. In the PA experiments the detected signal shape and amplitude might be affected by the laser beam's spot size and the energy density of the PA wave source [54].

The sedimentation process can be analyzed as a twolayer system. The upper layer consists of plasma or PBS and the lower layer of RBCs. The thickness of the upper layer increases as a function of time and the thickness of the lower layer decreases [20,29-31]. Heat and light transfer are complex in such systems [20,21]. The optical signal is converted to an acoustic pulse at the surface of the RBC/plasma layer. It is well known that this surface is not a pure reflecting surface [28,34]. It can be seen from Fig. 3 that the light intensity in the sample has a maximum at a certain depth. However, it must be noted that the optical absorption of red blood cells is almost two orders of magnitude higher than that of blood plasma and platelets [46]. Also, optical scattering is three or more orders of magnitude larger in red blood cells that in plasma and platelets [46] at the wavelength used-1064 nm. Hence, their effect on PA pulse generation is negligible.

Earlier research has demonstrated that the PA amplitude is dependent on the hematocrit [47]. Saha et al. found in simulations that the intensity of the PA signal increases when single RBCs form clusters [39]. The amplitude of the PA signal also increases when the RBC concentration of the illuminated area increases. Hence, those results support the findings in this paper. Aggregation affects scattering [34] and other optical properties. OCT is extensively studied in the context of different optical clearing agents [27-31]. The data in this paper show and support the fact that OCT can be used to monitor the sedimentation kinetics of various blood samples with different hematocrits.

\section{Conclusions}

The pulsed photoacoustic technique was demonstrated to be capable of detecting blood sedimentation dynamics in vitro. Photoacoustic pulse delay was found to be a good indicator for monitoring dextranaccelerated RBC sedimentation, both with diluted blood samples and with washed RBCs. Changes in the optical properties of the sample clearly affected acoustic signal generation in the sample and at the bottom of the cuvette.

\section{Acknowledgments}

This study was supported by the Academy of Finland (grant 128073). The author would like to also thank T. Sipola for help in preparation of the cuvette and Prof. A. V. Priezzhev, Dr. A. Bykov and Dr. Z. Zhao for help and fruitful discussions during the experiments. Final thanks are addressed to Oulu University Hospital, which provided their laboratory facilities for taking blood samples. 
Table 2 Sedimentation velocities calculated from PA and OCT signals and time intervals for the estimation of the sedimentation velocities.

\begin{tabular}{lllllll}
\hline Sample & $\begin{array}{l}\text { sample set } \mathbf{1}, \\
\text { velocity } \\
\text { estimation range }\end{array}$ & $\begin{array}{l}\text { sample set 1, } \\
\mathbf{P A} \\
\text { measurement } \\
(\mathbf{m m} / \mathbf{h})\end{array}$ & $\begin{array}{l}\text { sample set 2, } \\
\text { velocity estimation } \\
\text { range }\end{array}$ & $\begin{array}{l}\text { sample set 2, PA } \\
\text { measurement } \\
(\mathbf{m m} / \mathbf{h})\end{array}$ & $\begin{array}{l}\text { sample set 2, } \\
\text { velocity } \\
\text { estimation } \\
\text { range }\end{array}$ & $\begin{array}{l}\text { sample set 2, } \\
\text { OCT } \\
\text { measurement } \\
(\mathbf{m m} / \mathbf{h})\end{array}$ \\
\hline 1 & $0-30 \mathrm{~min}$ & $1.6 \pm 0.1$ & $0-30 \mathrm{~min}$ & $1.9 \pm 0.1$ & $5.3-16.2 \mathrm{~min}$ & $1.0 \pm 0.1$ \\
\hline 2 & $2-6 \mathrm{~min}$ & $26.7 \pm 1.0$ & $2-6 \mathrm{~min}$ & $38.4 \pm 0.7$ & $0.1-1.0 \mathrm{~min}$ & $15.3 \pm 0.1$ \\
\hline 3 & $0-30 \mathrm{~min}$ & $1.2 \pm 0.1$ & $0-30 \mathrm{~min}$ & $1.7 \pm 0.1$ & $5.0-15.1 \mathrm{~min}$ & $1.2 \pm 0.1$ \\
\hline 4 & $0-4 \mathrm{~min}$ & $39.5 \pm 0.8$ & $0-4 \mathrm{~min}$ & $40.7 \pm 2.8$ & $0.2-0.4 \mathrm{~min}$ & $29.9 \pm 0.1$ \\
\hline 5 & $0-30 \mathrm{~min}$ & $1.4 \pm 0.1$ & $0-30 \mathrm{~min}$ & $1.7 \pm 0.1$ & $10.5-21.0 \mathrm{~min}$ & $1.5 \pm 0.1$ \\
\hline 6 & $0-4 \mathrm{~min}$ & $39.5 \pm 2.1$ & $2-6 \mathrm{~min}$ & $32.6 \pm 0.9$ & $0.2-0.7 \mathrm{~min}$ & $19.5 \pm 0.1$ \\
\hline 7 & $0-30 \mathrm{~min}$ & $3.3 \pm 0.1$ & $0-30 \mathrm{~min}$ & $2.2 \pm 0.1$ & $5.6-9.4 \mathrm{~min}$ & $1.6 \pm 0.1$ \\
\hline 8 & - & - & $0-6 \mathrm{~min}$ & $35.7 \pm 0.1$ & $0.2-0.5 \mathrm{~min}$ & $32.6 \pm 0.1$ \\
\hline
\end{tabular}

